\title{
Epidemiología y susceptibilidad antifúngica de especies causantes de candidemia en la ciudad de Valdivia, Chile
}

\author{
Francisco Márquez, Isabel Iturrieta, Mario Calvo, Milton Urrutia y Patricio Godoy-Martínez
}

\section{Epidemiology and antifungal susceptibility of species producing candidemia in Valdivia, Chile}

Background: Candidemia disease in Chile is not very known, despite being a very common and serious disease. Aim: To analyze incidence and susceptibility profile of species from the genus Candida in Hospital Base Valdivia. Material and Methods: All candidemia cases presented between March 2009 and August 2011 in our institution were identified. Species distribution and susceptibility were studied through the disk diffusion method. Results: Twenty-seven candidemia episodes were studied. Incidence varied from 0.3 to 0.7 by a thousand discharges (according to clinical service). Risk factors were hospitalization, preliminary use of antibiotics, aged people and underlying disease (renal failure, heart and lung disease). The most frequent species was C. albicans, followed by C. tropicalis, C. glabrata and C. krusei. Voriconazole was the antifungal showing the best in vitro performance. Amphotericin B, fluconazole and itraconazole presented variable results. Discussion: Incidence found was superior to that described in developed countries. Candida albicans constitute the most frequent species, with high sensitivity levels to fluconazole; Candida no albicans species must be monitored. Developing new research on the topic appears as fundamental to detect potential epidemiological variations.

Key words: Candidemia, yeasts, epidemiology, susceptibility.

Palabras clave: Candidemia, levaduras, epidemiología, susceptibilidad.

\section{Editorial relacionada en pág. 429}

\section{Introducción}

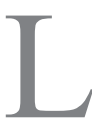

a sepsis producida por hongos, principalmente por especies del género Candida se ha transformado en un problema creciente durante la atención de salud de los pacientes en distintas partes del mundo ${ }^{1,2}$. Este hecho ha sido atribuido a un incremento de pacientes en riesgo de experimentar esta complicación, como son, una mayor cantidad de pacientes ingresados en unidades de cuidados intensivos, de los que cursan período postquirúrgico de grandes cirugías, de los pacientes que presentan neutropenia, la existencia de una mayor cantidad de pacientes inmunodeprimidos en general $\mathrm{y}$, por otra parte, una mejor sobrevida de los neonatos prematuros ${ }^{3,4}$.

La incidencia de candidemia, se ha incrementado considerablemente en las últimas décadas; de 2 casos por 1.000 ingresos el año (1996) alcanzó 2,4 casos por 1.000 ingresos el año 2003 en los Estados Unidos de América (E.U.A.), llegando a ser el cuarto agente más aislado en hemocultivos de hospitales norteamericanos ${ }^{3}$. La incidencia de candidemia en América Latina es variable; en un estudio realizado por Nucci y cols. ${ }^{5}$, en siete países, se observó una incidencia global 1,18 por 1.000 admisiones siendo los valores por país en orden decreciente de 1,95 en Argentina, 1,92 en Colombia, 1,72 en Venezuela, 1,38 en Brasil, Ecuador y Honduras con 0,9 y Chile con 0,335.

La frecuencia de aislamiento de las diferentes especies presenta variaciones significativas dependiendo de la ubicación geográfica. En todo el mundo, C. albicans sigue siendo la especie más aislada, pero entre las especies de Candida no albicans, se observan variaciones considerables, pues en E.U.A. y Europa existe un claro predominio de C. glabrata, en cambio, en América Latina los aislados más frecuentes son $C$. parapsilosis y $C$. tropicalis ${ }^{1,3,5,6}$.

La candidemia ha sido asociada con una alta tasa de mortalidad atribuida y global, especialmente en el grupo de pacientes críticos. Si bien, el interés en estas infecciones ha llevado al desarrollo de nuevos fármacos y estrategias para su manejo, la morbi-mortalidad de la candidemia se mantiene en cifras bastante elevadas cuando es comparada con la generada por otros cuadros infecciosos. Cifras recientes muestran que la tasa de mortalidad atribuida de candidemia ha sido estimada entre 25 y $38 \% \%^{7-9}$.

Debido a que en América Latina existen muy pocos estudios sobre la epidemiología de la candidemia, este trabajo tiene como objetivo presentar la incidencia, epidemiología y perfil de susceptibilidad de los agentes de candidemia identificados en un Hospital Regional del sur de Chile.
Universidad Santo Tomás, Sede Valdivia (FM). Universidad Austral de Chile. Instituto de Microbiología Clínica (II, PGM) Instituto de Medicina ((MC). Universidad de Antofagasta (MU).

Mario Calvo Arellano ha sido conferencista financiado por Pfizer y Merck, Sharp \& Dohme, sin ser empleado de estas empresas. Los autores manifiestan que no existen de manera directa o indirecta otros conflictos de interés.

Fuente de financiamiento Proyecto DID: 2010-70 Universidad Austral de Chile.

Recibido: 8 de agosto de 2016 Aceptado: 31 de agosto de 2017

Correspondencia a: Patricio Godoy-Martínez patricio.godoy@uach.cl 


\section{Material y Método}

El Hospital Base Valdivia (HBV) es el único Centro Asistencial de Alta Complejidad de la XIV Región de Los Ríos. Cuenta con 505 camas incluyendo Unidades de Pacientes Críticos (UPC) de adultos y pediátrica y tiene un carácter docente de pre y postgrado.

Los episodios de candidemia que se presentaron entre marzo de 2009 y agosto de 2011 fueron identificados a partir del registro de hemocultivos positivos provenientes de pacientes internados en el HBV. En caso de existir más de un aislamiento de Candida spp. en el mismo paciente, sólo el primer episodio de candidemia fue analizado. Para cada caso, se recopiló información bio-demográfica y factores de riesgo que presentaba el paciente, en forma retrospectiva, así como, la especie del agente identificado y el perfil de resistencia a los antifúngicos.

\section{Identificación fenotípica}

Las levaduras fueron aisladas en el sistema de hemocultivos automatizado BACTEC 860 , siendo posteriormente sembradas en medio CHROMagar Candida ${ }^{\mathbb{R}}$ e incubadas a $30^{\circ} \mathrm{C}$ durante $96 \mathrm{~h}$. La observación de las estructuras fúngicas fue posible a través del microcultivo en agar arroz Tween 80 donde se incubaron a $25-28^{\circ} \mathrm{C}$ durante 48 a 96 h. La identificación final fue realizada utilizando la metodología clásica de asimilación de carbohidratos; para las cepas que presentaron problemas con esta metodología fue utilizado el sistema ID-32C (bioMérieux Marcy I'Etoile, France) $^{10}$.

\section{Identificación molecular}

Se utilizó la técnica de amplificación aleatoria de ADN polimórfico (RAPD) utilizando la metodología descrita por Tavanti y cols. ${ }^{11}$, para la identificación molecular del complejo C. parapsilosis.

\section{Test de susceptibilidad antifúngica}

Fue evaluada la susceptibilidad in vitro frente a fluconazol (FLC), voriconazol (VOR), anfotericina B y

Tabla 1. Estimación de la incidencia de candidemia por mil egresos. Hospital Base Valdivia (2009-2011)

\begin{tabular}{llll}
\hline & $\mathbf{2 0 0 9}$ & $\mathbf{2 0 1 0}$ & $\mathbf{2 0 1 1}$ \\
Tiempo de seguimiento & 10 meses & 12 meses & 8 meses \\
Casos UCl & 3 & 4 & 1 \\
Casos No UCl & 5 & 11 & 3 \\
\hline Incidencia para ambos casos & 0,5 & 0,7 & 0,3 \\
Incidencia UCl & 2,1 & 2,2 & 0,9 \\
Incidencia No UCl & 0,3 & 0,6 & 0,3 \\
\hline
\end{tabular}

UCl: Unidad de Cuidados Intensivos. caspofungina utilizando el método de disco difusión con tabletas de Neo-Sensitabs ${ }^{\circledR}$ (Rosco, Denmark) en una concentración de $25 \mu \mathrm{g}$ de fluconazol, $1 \mu \mathrm{g}$ voriconazol, $10 \mu \mathrm{g}$ de anfotericina B y $5 \mu \mathrm{g}$ de caspofungina, en agar Mueller-Hinton (MHA) (BBL), suplementado con $2 \%$ de glucosa y $0,5 \mu \mathrm{g} / \mathrm{mL}$ de azul de metileno siguiendo el protocolo descrito en el documento M44-A del CLSI (2004) para fluconazol y voriconazol. Los diámetros de los halos fueron medidos en milímetros hasta el punto en que se observaba una reducción en el crecimiento ( $80 \%$ para los azoles). Los criterios para clasificar como susceptible (S), intermediario (I) o resistente (R) fueron los descritos por el fabricante: FLC (S $\geq 19 \mathrm{~mm}, \mathrm{I} 18 \sim 15 \mathrm{~mm}, \mathrm{R} \leq 14 \mathrm{~mm})$; $\operatorname{VOR}(\mathrm{S} \geq 17 \mathrm{~mm}, \mathrm{I} 16 \sim 14 \mathrm{~mm}, \mathrm{R} \leq 13 \mathrm{~mm})$; Fueron analizadas también las siguientes cepas: C. albicans (ATCC 90028), C. albicans (ATCC 28367), C. dubliniensis (CBS 7869) C. parapsilosis (ATCC 22019), C. orthopsilosis (ATCC 96141) y C. metapsilosis (ATCC 96143) para control de calidad. Una limitación de la metodología de disco difusión es que está validada solamente para los antifúngicos fluconazol y voriconazol.

\section{Datos para el cálculo de incidencia}

Se efectuó de acuerdo al número de casos de candidemia (hemocultivos) en los Servicios de UCI y otros Servicios en los años 2009, 2010 y 2011, en base a los egresos totales de las unidades de paciente crítico existentes en el HBV para los períodos estudiados.

\section{Factores de riesgo}

La caracterización de los factores de riesgo fue determinada mediante la revisión de los registros clínicos del HBV correspondientes a los pacientes estudiados.

\section{Resultados}

Un total de 27 episodios de candidemia fueron identificados en el período de estudio. La incidencia de candidemia varió entre 0,3 y 0,7 casos por 1.000 egresos considerando servicios UPC y no UPC y para los casos de UPC varió entre 2,2 y 0,9 casos por 1.000 egresos (Tabla 1). La información demográfica de los pacientes se describe en la Tabla 2.

El 70,4\% de los pacientes fueron del sexo femenino. El rango etario presentado por estos pacientes fue de 1 a 84 años, siendo mayor la incidencia en pacientes $>65$ años de edad (59,3\%).

\section{Co-morbilidades}

En la totalidad de los casos existía una o más enfermedades crónicas de base al momento de presentarse el cuadro de candidemia, dentro de los cuales $50 \%$ presentó insuficiencia renal, $43 \%$ presentó enfermedad cardiaca, 


\begin{tabular}{|c|c|c|c|c|c|c|}
\hline \multirow[t]{3}{*}{ Pacientes } & \multicolumn{6}{|c|}{ Especies de Candida } \\
\hline & \multirow{2}{*}{$\begin{array}{c}\text { Total } \\
\text { (n: 27) }\end{array}$} & \multirow{2}{*}{$\begin{array}{c}\text { Total } \\
100,0 \%\end{array}$} & \multicolumn{2}{|c|}{ C. albicans } & \multicolumn{2}{|c|}{ Candidas no albicans } \\
\hline & & & $(n: 16)$ & $59,3 \%$ & $(n: 11)$ & $40,7 \%$ \\
\hline \multicolumn{7}{|l|}{ Edad (años) } \\
\hline $\mathrm{RN}-14$ & 4 & $14,8 \%$ & 3 & $18,75 \%$ & 1 & $9,1 \%$ \\
\hline $15-49$ & 3 & $11,1 \%$ & 2 & $12,5 \%$ & 1 & $9,1 \%$ \\
\hline $50-65$ & 4 & $14,8 \%$ & 4 & $25,0 \%$ & 0 & $0,0 \%$ \\
\hline$>65$ & 16 & $59,3 \%$ & 7 & $43,75 \%$ & 9 & $81,8 \%$ \\
\hline \multicolumn{7}{|l|}{ Género } \\
\hline Masculino & 8 & $29,6 \%$ & 5 & $31,3 \%$ & 3 & $27,3 \%$ \\
\hline Femenino & 19 & $70,4 \%$ & 11 & $68,7 \%$ & 8 & $72,7 \%$ \\
\hline Hospitalización al momento del incidente de candidemia & 27 & $100,0 \%$ & 16 & $59,3 \%$ & 11 & $40,7 \%$ \\
\hline Uso de antimicrobianos de amplio espectro por más de 24 h en los 14 días previos & 27 & $100,0 \%$ & 16 & $59,3 \%$ & 11 & $40,7 \%$ \\
\hline \multicolumn{7}{|l|}{ Enfermedad de base n (\%) } \\
\hline Enfermedad crónica de base: & & & & & 1 & $3,6 \%$ \\
\hline Insuficiencia renal & 14 & $50,0 \%$ & 9 & $64,3 \%$ & 5 & $35,7 \%$ \\
\hline Enfermedad cardiaca & 12 & $42,9 \%$ & 8 & $66,7 \%$ & 4 & $33,3 \%$ \\
\hline Enfermedad pulmonar & 12 & $42,9 \%$ & 10 & $83,3 \%$ & 2 & $16,7 \%$ \\
\hline Diabetes mellitus tipo II & 8 & $28,6 \%$ & 6 & $75,0 \%$ & 2 & $25,0 \%$ \\
\hline Neoplasia & 5 & $17,9 \%$ & 4 & $80,0 \%$ & 1 & $20,0 \%$ \\
\hline Uso de catéter venoso central y/o periférico en las $24 \mathrm{~h}$ previas al incidente & 20 & $71,4 \%$ & 11 & $55,0 \%$ & 9 & $45,0 \%$ \\
\hline Supresor de acidez gástrica por más de 24 h en los 14 días previos & 18 & $64,3 \%$ & 14 & $77,8 \%$ & 4 & $22,2 \%$ \\
\hline Cirugía en los 3 meses previos & 14 & $50,0 \%$ & 8 & $57,1 \%$ & 6 & $42,9 \%$ \\
\hline Nutrición parenteral en las 24 h previas & 14 & $50,0 \%$ & 7 & $50,0 \%$ & 7 & $50,0 \%$ \\
\hline Tratamiento con corticoides por más de 24 h en los 14 días previos & 10 & $35,7 \%$ & 8 & $80,0 \%$ & 2 & $20,0 \%$ \\
\hline Estadía en UCl & 6 & $21,4 \%$ & 3 & $50,0 \%$ & 3 & $50,0 \%$ \\
\hline Ventilación mecánica en las 24 h previas & 4 & $14,3 \%$ & 3 & $75,0 \%$ & 1 & $25,0 \%$ \\
\hline Infección por VIH & 2 & $7,1 \%$ & 2 & $100,0 \%$ & 0 & $0,0 \%$ \\
\hline Trasplante en los 3 meses previos & 2 & $7,1 \%$ & 1 & $50,0 \%$ & 1 & $50,0 \%$ \\
\hline Tratamiento con inmunosupresores no corticoesteroidales & 2 & $7,1 \%$ & 1 & $50,0 \%$ & 1 & $50,0 \%$ \\
\hline Menor de 3 meses de edad & 2 & $7,1 \%$ & 1 & $50,0 \%$ & 1 & $50,0 \%$ \\
\hline Diálisis en las 72 h previas & 2 & $7,1 \%$ & 1 & $50,0 \%$ & 1 & $50,0 \%$ \\
\hline Quimioterapia por más de 24 h en los 14 días previos & 2 & $7,1 \%$ & 2 & $100,0 \%$ & 0 & $0,0 \%$ \\
\hline
\end{tabular}

$43 \%$ enfermedad pulmonar, $29 \%$ diabetes mellitus y $14 \%$ tenían neoplasia (Tabla 2).

\section{Factores de riesgo para candidemia}

La totalidad de los pacientes se encontraba hospitalizado al momento de presentación de la candidemia y todos habían recibido antibioterapia de amplio espectro en los 14 días previos. Otros factores de riesgo que se presentaron con menor frecuencia fueron: recepción de trasplante de órganos sólidos en los tres meses previos (7\%), tratamiento con fármacos inmunosupresores distintos a los corticosteroides ( $7 \%$ ), edad menor a 3 meses $(7 \%)$, tratamiento con terapia de sustitución renal en curso (7\%) y quimioterapia por un tiempo superior a $24 \mathrm{~h}$ en los 14 días previos al incidente candidemia (7\%) (Tabla 2).

\section{Distribución de las especies del género Candida}

Candida albicans fue la levadura más aislada con 16 cepas (59,3\%), seguida de C. tropicalis con 4 (14,8\%), C. glabrata $3(11,1 \%)$, C. krusei $2(7,4 \%)$, C. parapsilosis 1 $(3,7 \%)$ y $C$. guilliermondii $1(3,7 \%)$ (Tabla 3$)$.

\section{Susceptibilidad in vitro a antifúngicos}

Las cepas estudiadas tuvieron $100 \%$ de susceptibilidad a voriconazol y caspofungina. Anfotericina B presentó 90\% de actividad y solamente una cepa de C. krusei y una de $C$. parapsilosis mostraron perfiles de susceptibilidad dependiente de la dosis (SDD). Fluconazol presentó una buena actividad in vitro frente a C. albicans, C. tropicalis, C. parapsilosis y C. guilliermondii; no obstante, se observaron diferencias para C. glabrata y C. krusei 
Tabla 3. Perfiles se susceptibilidad de cepas de Candida sp. frente a anfotericina B, itraconazol, voriconazol y fluconazol. Hospital Base Valdivia. 2009-2011

\begin{tabular}{|c|c|c|c|c|c|c|c|c|c|c|c|c|c|c|c|c|}
\hline \multirow[t]{2}{*}{ Especie } & \multirow[b]{2}{*}{ n (\%) } & \multicolumn{3}{|c|}{ Anfotericina B } & \multicolumn{3}{|c|}{ Itraconazol } & \multicolumn{3}{|c|}{ Voriconazol } & \multicolumn{3}{|c|}{ Fluconazol } & \multicolumn{3}{|c|}{ Caspofungina } \\
\hline & & $\mathbf{S}$ & SDD & $\mathbf{R}$ & $\mathbf{S}$ & SDD & $\mathbf{R}$ & $\mathbf{S}$ & SDD & $\mathbf{R}$ & $\mathbf{S}$ & SDD & $\mathbf{R}$ & $\mathbf{S}$ & SDD & $\mathbf{R}$ \\
\hline C. albicans & $16(59,3)$ & $16(100)$ & $(0)$ & $0(0)$ & $(0)$ & $15(94)$ & $1(6)$ & $16(100)$ & $0(0)$ & $0(0)$ & $16(100)$ & (0) & $(0)$ & $16(100)$ & $0(0)$ & $0(0)$ \\
\hline C. tropicalis & $4(14,8)$ & $4(100)$ & (0) & $0(0)$ & (0) & $4(100)$ & $0(0)$ & $4(100)$ & $0(0)$ & $0(0)$ & $4(100)$ & (0) & (0) & $4(100)$ & $0(0)$ & $0(0)$ \\
\hline C. glabrata & $3(11,1)$ & $3(100)$ & $(0)$ & $0(0)$ & (0) & $3(100)$ & $0(0)$ & $3(100)$ & $0(0)$ & $0(0)$ & $0 \quad(0)$ & (0) & $3(100)$ & $3(100)$ & $0(0)$ & $0(0)$ \\
\hline C. krusei & $2(7,4)$ & (0) & $2(100)$ & $0(0)$ & $0 \quad(0)$ & $2(100)$ & $0(0)$ & $2(100)$ & $0(0)$ & $0(0)$ & $0 \quad(0)$ & $2(100)$ & (0) & $2(100)$ & $0(0)$ & $0(0)$ \\
\hline C. parapsilosis & $1 \quad(3,7)$ & $0 \quad(0)$ & $1(100)$ & $0(0)$ & $1(100)$ & $0 \quad(0)$ & $0(0)$ & $1(100)$ & $0(0)$ & $0(0)$ & $1(100)$ & $(0)$ & (0) & $1(100)$ & $0(0)$ & $0(0)$ \\
\hline C. guilliermondii & $1(3,7)$ & $1(100)$ & $(0)$ & $0(0)$ & $(0)$ & $1(100)$ & $0(0)$ & $1(100)$ & $0(0)$ & $0(0)$ & $1(100)$ & $(0)$ & (0) & $1(100)$ & $0(0)$ & $0(0)$ \\
\hline
\end{tabular}

las que mostraron SDD y resistencia, respectivamente, a este antifúngico. El antifúngico que presentó una menor actividad in vitro para todas las cepas analizadas fue itraconazol. Los perfiles de susceptibilidad de las diferentes especies se detallan en la Tabla 3.

\section{Discusión}

La candidemia es un problema de salud creciente universal $^{12}$; sin embargo, la importancia de la candidemia en los hospitales de Chile ha sido pobremente estudiada, dificultando el diseño de estrategias de diagnóstico y tratamiento apropiados. La incidencia en pacientes hospitalizados ha experimentado un incremento mundial progresivo en los últimos años ${ }^{12,13}$, fundamentalmente asociado al aumento en la sobrevida de pacientes críticos y pacientes afectados por una variedad de patologías debilitantes como neoplasias, trasplantes, procedimientos médicos invasores, infección por VIH/SIDA, tratamientos con inmunosupresores, entre otros, constituyendo una población de alto riesgo para el desarrollo de infecciones fúngicas, particularmente de candidemia ${ }^{14}$. Los datos obtenidos en nuestro hospital presentan una incidencia que varía desde 1,8 por 1.000 egresos en UCI a 0,4 por 1.000 egresos en las otras unidades, cifras similares a las presentadas por Marchetti y cols., en Suiza ${ }^{15}$ y superiores a los datos obtenidos por Tortorano y cols., en un estudio llevado a cabo en siete países de Europa ${ }^{16}$.

$\mathrm{Si}$ bien es cierto las tasas de incidencia descritas podrían ser mayores que en otros hospitales similares en magnitud, es importante considerar que el HBV es un centro de derivación de pacientes hematológicos procedentes de otras cuatro regiones del país, y no es menos cierto que ha habido cambios en el número de pacientes críticos manejados en este centro. Reflejo de esto ha sido, por ejemplo, el aumento del número de camas críticas de adultos en el HBV, que pasaron desde un total de 7 en el año 2002, todas de la Unidad de Cuidados Intensivos
(UCI), a 21 camas críticas (9 en UCI y 12 en la Unidad de Tratamiento Intermedio (UTI)). En el mismo período el país aumentó de 773 camas críticas (282 en UCI y 491 en UTI) a 1.004 (387 en UCI y 717 en UTI) ${ }^{17}$.

Se debe considerar, además, otros factores asociados a la condición de los pacientes hospitalizados en las Unidades Críticas, relacionados a los cambios de la población. En reportes de pacientes con distintas patologías en UCI chilenas, podemos notar también la gravedad actual de los pacientes. En el año 2004, el estudio nacional de sepsis mostró que 38,8\% de los pacientes hospitalizados en 64 UCIs a lo largo de todo el país, presentaban un cuadro de sepsis grave, con un APACHE II de 18,2 $\pm 6,4$, significativamente más alto que los pacientes sin esta condición (APACHE II 12,2 $\pm 7,3)^{18}$.

La mayoría de los casos de candidemia ocurrió en pacientes adultos sobre 65 años de edad (58\%) y hubo predominio en el sexo femenino $(68 \%)$ en relación al sexo masculino, consistente con lo descrito en otras regiones geográficas $^{13,20,21}$.

Llama la atención en nuestro grupo, que un bajo porcentaje de pacientes se encontraba en Unidades de Cuidados Intensivos (21\%), lo que contrasta con otras publicaciones $^{4,19,20}$. Por otra parte, la mitad de los pacientes se encontraba recibiendo nutrición parenteral y $71 \%$ tenía algún tipo de cateterización venosa, cifras similares a las descritas por Ajenjo y cols. ${ }^{13}$, en el año 2011, lo que apunta a que efectivamente se trata de pacientes de alta complejidad. Si bien es cierto, que el CVC no siempre es el origen de la infección, sí puede actuar como un reservorio, prolongando la duración del cuadro e incrementando, a su vez, el riesgo de desarrollo de focos metastásicos ${ }^{22}$.

Los principales hongos aislados de hemocultivos en el HBV correspondieron a C. albicans $(59,3 \%)$, C. tropicalis $(14,8 \%)$ y $C$. glabrata $(11,1 \%)$, distribución coincidente con la encontrada en el año 2011 en el Hospital Clínico de la Pontificia Universidad Católica de Chile ${ }^{13}$. Llama la atención que $C$. glabrata ocupe el tercer lugar, por sobre C. parapsilosis que ocupa el quinto lugar en nuestra 
investigación, ya que en América Latina C. parapsilosis es considerado el segundo o tercer agente en importancia $^{19,23,24}$ e incluso, hay reportes de ciertos países como México donde es descrita como el principal agente de candidemia ${ }^{25}$.

En relación a los perfiles de susceptibilidad presentados por las diferentes especies, fue posible constatar que la especie $C$. albicans efectivamente presenta altos niveles de sensibilidad a fluconazol $(82,1 \%)$, similar a lo ya descrito por otros autores ${ }^{13,25}$. Si bien es cierto, fluconazol es uno de los antifúngicos más utilizado en nuestro país, se debe tener en consideración la existencia de C. glabrata y C. krusei, frente a las cuales se observó un mayor nivel de resistencia, y es ahí donde radica la importancia de una identificación certera y oportuna del agente. Frente a anfotericina B no se observó resistencia, pero sí hubo cepas SDD de C. parapsilosis y C. krusei, por lo que voriconazol y caspofungina fueron los antifúngicos más efectivos, con susceptibilidad de todas las especies estudiadas.

La candidemia es considerada actualmente una condición de alta gravedad, con importantes tasas de morbilidad y mortalidad, fundamentalmente debido al tipo de pacientes que se ven afectados y los variados factores de riesgo asociados. El pronóstico de la candidemia mejora sustancialmente con la precocidad en el inicio de un tratamiento antifúngico adecuado y oportuno ${ }^{24}$. Sabemos que la epidemiología mundial ha experimentado importantes cambios, principalmente en la variación de la frecuencia relativa de especies, con un predominio de Candida no albicans; sin embargo, son pocos los estudios realizados en Chile que permitan conocer la epidemiología local y el comportamiento de las diferentes especies en términos de sus perfiles de susceptibilidad. El uso de azoles, específicamente fluconazol, ha sido uno de los factores con mayor influencia en el cambio de distribución de especies y el incremento de cepas resistentes ${ }^{26,27}$.

Es importante el desarrollo de nuevas investigaciones que permitan detectar potenciales variaciones y de esa forma orientar la toma de decisiones clínicas y gubernamentales de manera informada, teniendo como base la realidad local, que eventualmente pudiera no ser igual a la descrita en otras regiones geográficas. En este contexto, los datos expuestos confirman en nuestro centro, tanto la diversidad de las especies involucradas, como la presencia de distintos grupos afectados y la creciente resistencia a diferentes antifúngicos.

\section{Resumen}

Introducción: La epidemiología de la candidemia en Chile es poco conocida, a pesar de ser una enfermedad frecuente y grave. Objetivos: Analizar la incidencia y perfil de susceptibilidad in vitro de especies del género Candida causantes de candidemia en el Hospital Base Valdivia. Material y Métodos: Se identificó todos los casos de candidemia ocurridos en la institución entre marzo de 2009 y agosto de 2011. Se estudió la distribución de especies y susceptibilidad in vitro por el método de disco difusión. Resultados: Se estudiaron 27 episodios de candidemia. La incidencia varió de 0,3 a 0,7 por 1.000 egresos (según servicio clínico). Fueron factores de riesgo: la hospitalización, uso previo de antimicrobianos, edad avanzada y enfermedad de base (insuficiencia renal, enfermedad cardiaca y pulmonar). La especie más frecuente fue $C$. albicans seguida de C. tropicalis, C. glabrata y C. krusei. Voriconazol fue el antifúngico con mejor actividad in vitro y hubo resultados variables para anfotericina B, fluconazol e itraconazol. Discusión: La incidencia encontrada fue superior a la de países desarrollados. Candida albicans, constituye la especie más frecuente, con alta sensibilidad in vitro a fluconazol, debiendo ser vigiladas las especies de Candida no albicans. Es fundamental desarrollar nuevas investigaciones para detectar potenciales variaciones epidemiológicas.

\section{Referencias bibliográficas}

1.- Colombo A L, Guimarães T. Epidemiology of hematogenous infections due to Candida spp. Rev Soc Bras Med Trop 2003; 36 (5): 599-607.

2.- Costa J, Gadelha M. Capítulo 25: Candidíase. Costa J, Gadelha M, editores. Micologia médica à luz de autores contemporâneos, $1^{\mathrm{a}}$ edição. Rio de Janeiro: Ed. Guanabara Koogan S.A; 2004, p. 265-74.

3.- Pfaller M A, Diekema D J. Epidemiology of invasive candidiasis: a persistent public health problem. Clin Microbiol Rev 2007; 20: 133-63.

4.- Bouza E, Muñoz P. Epidemiology of candidemia in intensive care units. Int $\mathrm{J}$
Antimicrob Agents 2008; 32 (2): S87-91.

5.- Nucci M, Queiroz-Telles F, Alvarado-Matute T, Cortés J, Zurita J, Guzmán. Blanco M, et al. Epidemiology of candidemia in Latin America: A laboratory-based survey. PLoS ONE 2013; 8 (3): e59373.

6.- Hajjeh R, Sofair A, Harrison L, Marshall G, Arthington-Skaggs B, Mirza S, et al. Incidence of bloodstream infections due to Candida species and in vitro susceptibilities of isolates collected from 1998 to 2000 in a populationbased active surveillance program. J Clin Microbiol 2004; 42 (4): 1519-27.

7.- Zaragoza R, Pemán J. The diagnostic and therapeutic approach to fungal infections in critical care settings. Adv Sepsis 2008; 6: 90-8.

8.- Gómez J, García-Vázquez E, Espinosa C, Ruiz J, Canteras M, Hernández-Torres A, et al. Nosocomial candidemia at a general hospital: the change of epidemiological and clinical characteristics. A comparative study of 2 cohorts (1993-1998 versus 2002-2005). Rev Iberoam Micol 2009; 26 (3): 184-8.

9.- Ma C F, Li F Q, Shi L N, Hu Y A, Wang Y, Huang M, et al. Surveillance study of species distribution, antifungal susceptibility and mortality of nosocomial candidemia in a tertiary care hospital in China. BMC Infect Dis 2013; 22 (13): 337. doi: 10.1186/1471-233413-337. 
10.- De Freitas A R, Baeza L C, Faria M G, Dota K F, Godoy Martínez P, Svidzinski T I. Yeasts isolated from nosocomial urinary infections: antifungal susceptibility and biofilm production. Rev Iberoam Micol 2014; 31 (2): 104-8.

11.- Tavanti A, Davidson A D, Gow N A, Maiden M C, Odds F C. Candida orthopsilosis and Candida metapsilosis spp. nov. to replace Candida parapsilosis groups II and III. J Clin Microbiol 2005; 43 (1): 284-92.

12.- Sandven P. Epidemiology of candidemia. Rev Iberoam Micol 2000; 17 (3): 73-81.

13.- Ajenjo H, Aquevedo S A, Guzmán D A M, Poggi M H, Calvo A M, Castillo V C, et al. Epidemiologial profile of invasive candidiasis in intensive care units at a university hospital. Rev Chilena Infectol 2011; 28 (2): 118-22.

14.- Horn D L, Neofytos D, Anaissie E J, Fishman J A, Steinbach W J, Olyaei A J, et al. Epidemiology and outcomes of candidemia in 2019 patients: Data from the Prospective Antifungal Therapy Alliance Registry. Clin Infect Dis 2009; 48: 1695-703.

15.- Marchetti O, Bille J, Fluckiger U, Eggimann P, Ruef C, Garbino J, et al. Fungal Infection Network of Switzerland. Epidemiology of candidemia in Swiss tertiary care hospitals: secular trends, 1991-2000. Clin Infect Dis 2004; 38 (3): 311-20.

16.- Tortorano A, Peman J, Bernhardt H, Klingspor L, Kibbler C, Faure O, et al. Epidemiology of candidaemia in Europe: results of 28-month European Confederation of Medical Mycology (ECMM) hospital-based surveillance study. Eur
J Clin Microbiol Infect Dis 2004; 23 (4): 317 22.

17.- Gálvez S, González H, Labarca E, Cornejo R, Bruhn A, Ugarte H, et al. Medicina Intensiva en Chile: desafíos para su desarrollo. Documento de la Comisión Nacional de Medicina Intensiva del Ministerio de Salud. Rev Med Chile 2013; 141: 90-4.

18.- Dougnac A L, Mercado M F, Cornejo R R, Cariaga M V, Hernández G P, Andresen M H, et al y Grupo Chileno del Estudio de la Sepsis. Prevalencia de sepsis grave en las Unidades de Cuidado Intensivo. Primer estudio nacional multicéntrico. Rev Med Chile 2007; 135: 62030.

19.- Colombo A, Nucci M, Park B, Nouér S, Arthington-Skaggs B, da Matta D, et al. Epidemiology of candidemia in Brazil: a nationwide sentinel surveillance of candidemia in eleven medical centers. J Clin Microbiol 2006; 44 (8): 2816-23.

20.- Asmundsdottir L R, Erlendsdottir H, Gottfredsson M. Nationwide study of candidemia, antifungal use, and antifungal drug resistance in Iceland, 2000 to 2011. J Clin Microbiol 2013; 51 (3): 841-8.

21.- Diekema D J, Messer S A, Brueggemann A B, Coffman S L, Doern G V, Herwaldt L A, et al. Epidemiology of candidemia: 3-year results from the emerging infections and the epidemiology of Iowa organisms study. J Clin Microbiol 2002; 40 (4): 1298-302.

22.- Kullberg B J, Verweij P E, Akova M, Arendrup M C, Bille J, Calandra T, et al. European expert opinion on the management of invasive candidiasis in adults. Clin Microbiol Infect 2011; 17 (5): 1-12.

23.- Santos P E, Córdoba S, Carrillo-Muñoz A, Rodero L, Rubeglio E, Soria M. Epidemiology of fungaemia in a paediatric hospital of high complexity. Rev Iberoam Micol 2010; 27 (4): 200-2.

24.- Colombo A L, Guimarães T, Camargo LF, Richtmann R, Queiroz-Telles F, Salles M J, et al. Brazilian guidelines for the management of candidiasis-a joint meeting report of three medical societies: Sociedade Brasileira de Infectologia, Sociedade Paulista de Infectologia and Sociedade Brasileira de Medicina Tropical. Braz J Infect Dis 2013; 17 (3): 283-312.

25.- González G M, Elizondo M, Ayala J. Trends in species distribution and susceptibility of bloodstream isolates of Candida collected in Monterrey, Mexico, to seven antifungal agents: Results of a 3-year (2004 to 2007) surveillance study. J Clin Microbiol 2008; 46 (9): 2902-5.

26.- Maldonado N A, Cano L E, De Bedout C, Arbeláez C A, Roncancio G, Tabares A M, et al. Grupo GERMEN. Association of clinical and demographic factors in invasive candidiasis caused by fluconazole-resistant Candida species: a study in 15 hospitals, Medellín, Colombia 2010-2011. Diagn Microbiol Infect Dis 2014; 79 (2): 280-6.

27.- Liao X, Qiu H, Li R, Guo F, Liu W, Kang M, et al. China-SCAN Team. Risk factors for fluconazole-resistant invasive candidiasis in intensive care unit patients: An analysis from the China survey of candidiasis study. J Crit Care 2015; 30 (4): 862-5. 